\title{
Hematopoietic stem cell transplantation for the management of follicular lymphoma
}

This article was published in the following Dove Press journal:

Stem Cells and Cloning:Advances and Applications

30 April 2010

Number of times this article has been viewed

\author{
Chitra Hosing \\ Department of Stem Cell \\ Transplantation and Cellular Therapy, \\ The University of Texas M.D. \\ Anderson Cancer Center, Houston, \\ Texas, USA
}

\begin{abstract}
Although much has been published on the application of autologous and allogeneic hematopoietic stem cell transplantation in patients with follicular lymphoma (FL), no uniform consensus exists among physicians on when to use this strategy. Three large randomized trials failed to show a survival benefit using autologous transplantation for FL patients in first complete remission. Similarly, many Phase II or registry-based studies have also failed to show a survival benefit with autologous transplantation in relapsed or refractory FL patients, although the progression-free survival seems to be prolonged in transplant recipients. Allogeneic stem cell transplantation can cure a subset of patients with FL, but high nonrelapse mortality and morbidity remain a concern. No consensus exists on what conditioning regimen should be used, or how the newer monoclonal antibodies should be incorporated into the transplant paradigm. Here we present a review of the role of autologous and allogeneic hematopoietic stem cell transplantation in patients with FL.
\end{abstract}

Keywords: review, follicular lymphoma, allogeneic transplantation, autologous transplantation

\section{Background}

With approximately 15,000 new cases each year in the US, follicular lymphoma (FL) is the second most common subtype of non-Hodgkin's lymphoma (NHL). ${ }^{1}$ Advanced FL has traditionally been considered incurable with conventional treatment. ${ }^{2}$ In most cases, FL is characterized by a long median survival but a continuous pattern of relapse. ${ }^{3}$ Eventually the lymphoma becomes resistant to chemotherapy or undergoes transformation to the more aggressive large-cell histology.

In the last five years, several institutions in the US have reported an improvement in the survival of FL patients. ${ }^{4,5}$ This improvement is due in part to the availability of drugs like rituximab ${ }^{6,7}$ and to radioimmunoconjugates, ${ }^{8}$ which have resulted in higher response rates, longer times to disease progression, and due to better supportive care.

High-dose chemotherapy (HDT) supported by both autologous and allogeneic hematopoietic stem cell transplantation (HSCT) has been studied extensively in patients with FL. However, at present, there is no consensus among physicians on when to use this strategy. Considerable evidence suggests that HDT alone may not be effective in eradicating FL. However, in some studies, a plateau in progression-free survival (PFS) has been observed which could suggest that a subgroup of patients with FL might be cured by autologous HSCT. ${ }^{9}$ Addition of monoclonal antibodies like rituximab and radioimmunoconjugates to the transplant regimen may further improve 
the outcomes. ${ }^{10,11}$ Monoclonal antibodies have also been used during hematopoietic stem cell mobilization to obtain "tumor-free" grafts. ${ }^{12}$

In allogeneic HSCT, donor cells repopulate the marrow, and a graft-versus-lymphoma (GVL) effect may also play an important role in preventing relapses. ${ }^{13-15}$ Observations that support the presence of a GVL effect are the induction of complete remission (CR) by modulating immunosuppressive therapy and by infusing donor lymphocytes into patients whose FL relapses after allogeneic HSCT. ${ }^{16-18}$

Observations in FL patients that autologous HSCT is associated with a low transplant-related mortality (TRM) but with higher relapse rates compared with allogeneic HSCT $^{19,20}$ led to the concept of using nonmyeloablative or reduced-intensity conditioning regimens (RIC, also called minitransplants) for allogeneic transplantation. ${ }^{21}$ The preparative regimen itself is not effective in eradicating FL, but it is immunosuppressive enough to allow engraftment of allogeneic peripheral blood stem cells (PBSC) or a bone marrow graft. In vivo expansion of immunocompetent cells after successful engraftment is responsible for the GVL effect. The less intensive preparative regimen is associated with a lower TRM and can be used in FL patients who are not suitable for myeloablative regimens because of advanced age or comorbidities. Also, the risk of acute severe graftversus-host disease (GVHD) may be lower in these patients because development of GVHD is related in part to the toxicity of the conditioning regimen and subsequent cytokine production. $^{22,23}$ The GVL effect may be augmented by the infusion of additional donor lymphocytes in FL patients who achieve successful engraftment. ${ }^{24}$

In recent years, allogeneic transplants have become safer, with TRM reduced from $40 \%$ to $10 \%$ or less at one year posttransplant. ${ }^{25,26}$ These improvements are attributable to better patient selection, better supportive care measures, improved human leukocyte antigen typing, advances in prevention and management of acute and chronic GVHD, and introduction of RIC and nonmyeloablative transplant regimens.

Although several published trials have evaluated the role of HDT and allogeneic or autologous HSCT in NHL, interpretation of these results is hindered by low patient enrolments, differences between the trials in selection criteria and transplant regimens used, and relatively short follow-up. It is also important to remember that the patient populations studied in these trials were a highly selected group and thus the results may not be applicable to all patients with FL. ${ }^{27}$

\section{Autologous transplantation}

HDT with autologous bone marrow or PBSC transplantation for patients with relapsed or recurrent FL has produced disease-free survival (DFS) rates from $31 \%$ to $63 \%$ and overall survival (OS) rates from $50 \%$ to $69 \%$ at two to eight years of follow-up. ${ }^{28-30}$ For patients who underwent transplant in first remission, DFS rates vary from $38 \%$ to $65 \%$, and OS rates of up to $75 \%$ at three to 10 years of follow-up have been reported (Table 1). 9,31,32 The differences in outcomes may be related to various factors, including the small number of patients in each study, selection criteria, patient characteristics, source of stem cells, purging, preparative regimen used, timing of transplantation, and posttransplant maintenance. Many of these studies included patients with indolent lymphoma histologies rather than only patients with FL histology.

\section{Autologous transplantation for relapsed or refractory $\mathrm{FL}$}

Numerous Phase II studies have evaluated the role of autologous HSCT in patients with relapsed or refractory FL. ${ }^{28-30,33}$ Rohatiner et al published mature data from a retrospective analysis of HDT supported by autologous bone marrow transplantation as consolidation therapy for second or subsequent remission in patients with $\mathrm{FL} .{ }^{28} \mathrm{~A}$ total of 121 patients received cyclophosphamide and total body irradiation (TBI) supported by autologous bone marrow transplantation. The graft mononuclear cell fraction was treated ex vivo with anti-CD20 monoclonal antibodies and complement. Of interest was an apparent plateau in the remission duration curve of $48 \%$ at 12 years. The 10 -year OS and PFS rates were 54\% and 48\%, respectively. Both remission and OS durations were significantly longer in patients treated in a second remission than in an agematched, remission-matched group of patients who had been treated at the same institution before the introduction of autologous HSCT. However, the development of secondary myelodysplasia (s-MDS) and secondary acute myeloblastic leukemia (s-AML) resulted in 15 deaths $(12.4 \%)$ in the transplanted patients. ${ }^{28}$

A retrospective study by Montoto et al evaluated the outcomes of 693 patients with FL who had undergone HDT. ${ }^{33}$ Of the 693 patients, 131 were in first CR. Median follow-up was 10.3 years. The 10- and 15-year PFS for the entire series were $31 \%$ and $27 \%$, respectively. On multivariate analysis, younger age and disease status at the time of HSCT correlated with longer PFS. Ten-year and 15-year OS rates with HDT were $52 \%$ and $47 \%$, respectively. On multivariate analysis, shorter OS was associated with older 
Table I Autologous hematopoietic stem cell transplantation for follicular lymphoma

\begin{tabular}{|c|c|c|c|c|c|}
\hline Trial & Patients (n) & Preparatory regimen & Follow-up (years) & PFS/EFS (\%) & OS (\%) \\
\hline \multicolumn{6}{|c|}{ Relapsed/recurrent disease/primary induction failure } \\
\hline Rohatiner $^{28}$ & $12 \mid$ & $\mathrm{Cy} / \mathrm{TBI}$ & 9 & 48 at $10 y$ & 54 at $10 y$ \\
\hline Freedman ${ }^{29}$ & 153 & $\mathrm{Cy} / \mathrm{TBI}$ & 5 & 42 at $8 y$ & 66 at $8 y$ \\
\hline \multirow[t]{2}{*}{ Bierman $^{30}$} & 100 & TBI 77 & 2.6 & 44 at $4 y$ & 65 at $4 y$ \\
\hline & & Non-TBI 23 & & & \\
\hline Apostolidis $^{36}$ & 99 & $\mathrm{Cy} / \mathrm{TBI}$ & 5.5 & 63 at $5 y$ & 69 at $5 y$ \\
\hline \multirow[t]{3}{*}{$\mathrm{Cao}^{37}$} & 49 (FLGL) & TBI 60 & - & 44 at $4 y$ & 60 at $4 y$ \\
\hline & 26 (FLCL) & Non-TBI 32 & & $5 \mathrm{I}$ at $4 y$ & 58 at $4 y$ \\
\hline & 17 (TFL) & & & 49 at $4 y$ & 50 at $4 y$ \\
\hline \multicolumn{6}{|c|}{ First remission (randomized) } \\
\hline \multirow[t]{2}{*}{ Lenz $^{32}$} & 240 & HSCT & 4.2 & 65 at $5 y$ & 84 at 5 y overall \\
\hline & & chemo & & $\begin{array}{l}33 \text { at } 5 y \\
(P<0.000 \mathrm{I})\end{array}$ & \\
\hline \multirow[t]{3}{*}{ Gyan? } & 172 & HSCT & 9 & 64 at $9 y$ & 76 at $9 y$ \\
\hline & & chemo & & 39 at $9 y$ & 80 at $9 y$ \\
\hline & & & & $(P=0.004)$ & $(P=N S)$ \\
\hline \multirow[t]{3}{*}{ Sebban ${ }^{40}$} & 401 & HSCT & 7.5 & 38 at $7 y$ & 71 at $7 y$ \\
\hline & & chemo & & 28 at $7 y$ & 76 at $7 y$ \\
\hline & & & & $(P=N S)$ & $(P=\mathrm{NS})$ \\
\hline \multicolumn{6}{|c|}{ First remission and relapsed refractory } \\
\hline \multirow[t]{2}{*}{ Montoto ${ }^{33}$} & CRI $|3|$ & TBI-based 40I & 10.3 & 27 at $15 y$ & 47 at 15 y \\
\hline & Beyond CRI 562 & Chemo-based 289 & & & \\
\hline
\end{tabular}

Abbreviations: HSCT, hematopoietic stem cell transplantation; Cy/TBI, cyclophosphamide/total body irradiation; PFS, progression-free survival; EFS, event-free survival; OS, overall survival; FLGL, follicular low-grade lymphoma; FLCL, follicular large cell lymphoma; TFL, transformed follicular lymphoma; TBI, total body irradiation; NS, not significant; $C R$, complete remission; $y$, years.

age, chemoresistant disease, stem cells derived from bone marrow, and TBI-containing regimens. Thirty-nine patients developed s-MDS/s-AML, and 34 of these 39 patients had received TBI as the conditioning regimen. The 10 -year nonrelapse mortality (NRM) was $6 \%$ for those who received nonTBI-based regimens and $16 \%$ for those receiving TBI-based regimens. On multivariate analysis, older age, chemoresistant disease, and TBI were associated with a higher NRM. As in the previous study, there was a plateau in the PFS curve, again suggesting that a select group of patients might be cured with HDT. In this analysis, TBI-containing regimens were associated with a negative impact on survival. ${ }^{33}$

In a different study from the Dana Farber Cancer Institute, 153 patients with relapsed FL received monoclonal antibody-purged autologous bone marrow transplantation and showed DFS and OS rates at eight years of $42 \%$ and $66 \%$, respectively. ${ }^{29}$ Patients whose bone marrow was negative as assessed by polymerase chain reaction (PCR) for bcl-2/IgH gene rearrangement after purging experienced longer freedom from recurrence than did those whose bone marrow remained PCR-positive $(P<0.0001)$. Continued PCR negativity in follow-up bone marrow samples was also strongly predictive of continued CR. The 12-year survival from diagnosis for these 153 patients was an impressive $69 \% .{ }^{29}$ Bierman et al presented a retrospective analysis of 100 patients who had undergone autologous HSCT for FL at their institution between 1983 and 1993. ${ }^{30}$ The median follow-up duration of surviving patients was 2.6 years (range 1.0-11.7). The OS rate at four years was $65 \%$ and the relapse-free survival (RFS) rate was estimated to be $44 \%$. Bierman et al did not observe a definite plateau in the RFS curve. The only factor that was significantly associated with OS and RFS was the number of chemotherapy regimens administered prior to transplantation. ${ }^{30}$

The prospective CUP trial was initiated to analyze the value of ex vivo purging in patients with relapsed chemosensitive FL who were undergoing HDT and autologous HSCT. ${ }^{34,35}$ After three cycles of chemotherapy, responsive patients were randomized to three more cycles of the same chemotherapy (C), or HDT followed by autologous unpurged (U) or purged (P) HSCT. Purging was performed using a cocktail of monoclonal antibodies. Of the 140 patients registered, 89 fulfilled the criteria for randomization (C: 24 , U: 33, and P: 32). Median follow-up time was 69 months in surviving patients. Patients in the $\mathrm{U}$ and $\mathrm{P}$ arms had higher PFS/RFS rates. Kaplan-Meier estimates of two-year PFS for $\mathrm{C}$, $\mathrm{U}$, and $\mathrm{P}$ were $26 \%, 58 \%$, and $55 \%$, respectively (hazards ratio for $\mathrm{C}$ versus $\mathrm{U}+\mathrm{P}$ was $0.3, P=0.0009)$. OS rates at four years for $\mathrm{C}, \mathrm{U}$, and $\mathrm{P}$ were $46 \%, 71 \%$, and $77 \%$, respectively (hazards ratio for $\mathrm{C}$ versus $\mathrm{U}+\mathrm{P}$ was $0.4, P=0.026$ ). Unfortunately, the trial was terminated early due to slow patient 
accrual, and therefore the role of ex vivo purging could not be evaluated. ${ }^{34,35}$

To evaluate the long-term results of HDT in FL with specific emphasis on the prognostic significance of PCRdetectable $\mathrm{Bcl}-2 / \mathrm{IgH}$ rearrangements, Apostolidis et al treated $99 \mathrm{FL}$ patients with HDT as consolidation therapy for second or subsequent remission. ${ }^{36}$ In vitro purging of the bone marrow graft was accomplished by treatment with anti-B-cell antibodies and complement. Median follow-up was 5.5 years (range 1.5-12.5). Overall, $12 \%$ of patients developed s-MDS or s-AML. Kaplan-Meier estimates of freedom from recurrence and survival rates at five years were $63 \%$ and $69 \%$, respectively. Multivariate analysis showed that absence of the Bcl-2/IgH rearrangement at the time of diagnosis and three or fewer treatment episodes before HDT were significant prognostic factors for improved survival. For patients bearing Bcl-2/IgH rearrangements in univariate and multivariate analyses, absence of a PCRdetectable Bcl-2/IgH rearrangement during follow-up was associated with a significantly lower risk of recurrence $(P<0.001)$ and death $(P=0.02)$, whereas the PCR status of the reinfused bone marrow graft did not correlate with outcome. ${ }^{36}$

In another retrospective analysis, Cao et al investigated the outcome of HDT and autologous HSCT in patients with FL beyond first remission. ${ }^{37}$ Ninety-two patients with primary induction failure or with relapsed FL were treated with myeloablative therapy followed by transplantation of purged autologous bone marrow or PBSC. The four-year estimate of OS was $60 \%$ and of DFS was $44 \%$ in patients with follicular low-grade histology. A TBI-containing HDT regimen was associated with significantly longer DFS and OS in multivariate analysis, and OS was also significantly longer among those who had received three or fewer chemotherapy regimens prior to transplantation. In a subset analysis of 17 patients with transformed FL, the four-year estimated rate of OS was $50 \%$ and of DFS was $49 \%$. There were three occurrences of s-MDS that yielded an estimated incidence rate of $7 \%$ at 56 months. Contrary to the results obtained by Montoto et al in this study, the TBIcontaining regimen appeared to be superior to the BCNU (carmustine)-based regimen for relapsed FL, although late effects could not be ascertained because of the short duration of follow-up. ${ }^{33,37}$

With the availability of rituximab, in vivo purging strategies have largely replaced in vitro purging methods. ${ }^{11,38,39}$ Using rituximab and high-dose cytarabine followed by granulocyte-colony stimulating factor in 14 patients with relapsed or refractory FL or mantle cell lymphoma enrolled in a program of HDT and autologous HSCT allowed for the collection of a PBSC graft that was free of monoclonal B-cells and contained an adequate cell dose. Of nine patients PCR-positive for bcl-2 or bcl-1 in blood and marrow at the start of immunochemotherapy, all showed PCR-negative PBSC grafts. The widespread use of immunochemotherapy administered prior to mobilization also produces B-cell depletion and may also play a role in the collection of tumor-free grafts. Tarella et al reported on the impact of adding rituximab to a modified high-dose sequential chemotherapy regimen in patients with NHL. ${ }^{11}$ Included in the study were 223 patients with FL who received the original or a modified high-dose sequential chemotherapy regimen. Two parameters significantly influenced the outcome of multivariate analysis, ie, disease status at HDT and the addition of rituximab. In those who received rituximab and those who did not receive it, the five-year OS rates were $82 \%$ and $68 \%(P=0.01)$ and eventfree survival (EFS) rates were $66 \%$ and $46 \%(P=0.001)$, respectively. ${ }^{11}$

Sebban et al evaluated the impact of HDT and HSCT and rituximab on the outcome for patients with relapsed FL. Two cohorts of patients treated in two successive randomized Groupe d'Etude des Lymphomes de l'Adulte (GELA) studies, (Groupe d'Etude des Lymphomes Folliculaires [GELF] GELF-86 and GELF-94) with the same chemotherapy regimen were studied. ${ }^{40,41}$ Of the 364 patients included in these two studies, 254 had FL that progressed or relapsed and were included in the analysis. Of the 254 patients, 98 were treated with HDT, including 33 with a rituximabcontaining salvage regimen and 69 with rituximab alone or combined with chemotherapy but without HDT. Patient characteristics at diagnosis were similar in all subgroups. HDT was associated with a statistically significant benefit of EFS for relapse and survival after relapse. Rituximab was associated with a greater benefit than HDT for these two endpoints. When both treatments were combined, patients treated with the rituximab-containing salvage regimen followed by HDT after relapse had a five-year survival rate of more than $90 \% .^{40,41}$

Some of the factors that have been found to be statistically significant for DFS rate and/or OS after autologous HSCT for relapsed or refractory FL are patient age at the time of transplantation, ${ }^{30,33}$ presence of minimal residual disease before and after transplantation, ${ }^{29,36}$ chemotherapyresistant versus chemotherapy-sensitive disease at the time of transplantation, ${ }^{30,33}$ number of chemotherapy regimens or 
treatment episodes prior to transplantation, ${ }^{33,37,42}$ and the type of conditioning regimen..$^{33,37}$

\section{Autologous transplantation in first complete remission of FL}

Good results have also been reported with HDT and autologous HSCT in patients with FL in first remission (Table 1). 9,32,40 The German Low-Grade Lymphoma Study Group (GLSG) initiated a randomized trial to compare the effect of myeloablative radiochemotherapy followed by autologous HSCT with interferon-alpha (IFN- $\alpha$ ) maintenance therapy for patients with FL in first remission. ${ }^{32} \mathrm{~A}$ total of 307 patients were enrolled. After two cycles of induction chemotherapy, patients were randomly assigned to either the HSCT or the IFN- $\alpha$ group. Two hundred and forty patients were evaluable for the comparison of HSCT and IFN- $\alpha$. The five-year PFS rate was significantly longer in the HSCT arm (65\% versus $33 \%$ in the HSCT and IFN- $\alpha$ arms, respectively, $P<0.0001)$. Because of the short follow-up, the impact on OS could not be determined. ${ }^{32}$

The Groupe Ouest Est d'Etude des Leucémies et Autres Maladies du Sang (GOELAMS) multicenter study randomized 172 patients with untreated FL to either immunochemotherapy or HDT followed by purged HSCT. ${ }^{9}$ The conditioning regimen for HSCT was TBI and cyclophosphamide. The nine-year OS was similar in the HDT and conventional chemotherapy groups ( $76 \%$ and $80 \%$, respectively). Again the PFS was significantly higher in the HSCT than in the chemotherapy group (nine-year PFS 64\% versus 39\%; $P=0.004$ ). A PFS plateau was observed in the HDT group after seven years. On multivariate analysis, OS and PFS were affected independently by performance status score, number of nodal areas involved, and treatment group. Secondary malignancies occurred more frequently in the HDT group than in the chemotherapy group. The occurrence of a PFS plateau is encouraging and may suggest that a subgroup of patients with FL might be cured with HSCT. ${ }^{9}$

Sebban et al compared a standard chemotherapy regimen + interferon with standard chemotherapy followed by HDT + ASCT in treatment-naive patients with advanced FL. ${ }^{40}$ Of a total of 401 patients were enrolled, 209 patients received chemotherapy and IFN- $\alpha$ for 18 months and 192 received chemotherapy followed by HDT and autologous HSCT with a TBI-containing regimen. Overall response rates were similar in both groups $(79 \%$ and $78 \%$ after induction chemotherapy). Of the 150 patients eligible for HDT, 131 underwent transplantation. Intent-to-treat analysis after a median follow-up of 7.5 years showed no difference between the two arms for OS $(P=0.53)$ or $\operatorname{EFS}(P=0.11)$. Patients with a complete response at the end of the induction therapy had a statistically longer EFS and OS $(P=0.02$ and $P<0.001$, respectively). ${ }^{40}$

In a randomized, multicenter study by Ledatto et al patients received chemotherapy followed by rituximab or rituximab-supplemented high-dose sequential chemotherapy with autologous HSCT to assess the value of intensified chemotherapy as a first-line treatment for high-risk FL. ${ }^{43}$ After a median follow-up of 51 months, the four-year EFS was $28 \%$ and $61 \%$, respectively, in the chemotherapy versus HSCT $\operatorname{arm}(P<0.001)$, with no difference in OS. Molecular remission was achieved in $44 \%$ of chemotherapy patients and in $80 \%$ of patients receiving HSCT $(P<0.001)$ and was the strongest independent outcome predictor. ${ }^{43}$

With the exception of the study by Ledatto et al all studies mentioned were conducted in the pre-rituximab era. Therefore, the impact of rituximab cannot be fully evaluated in patients undergoing autologous HSCT. Two recent abstracts have addressed the role of autologous HSCT in patients with FL who have received front-line rituximab therapy. ${ }^{44,45}$ Hiddemann et al conducted a retrospective analysis of two GLSG studies, which showed that rituximab-cyclophosphamide/doxorubicin/ vincristine/prednisone (R-CHOP) followed by IFN maintenance achieved a five-year PFS of $67 \%$ and was comparable with CHOP followed by autologous HSCT. ${ }^{45}$ R-CHOP followed by HSCT, however, revealed a five-year PFS of 79\%, with only one relapse after 24 months. This study suggested that autologous HSCT may have a role in the era of front-line R-CHOP therapy, particularly for intermediate- or high-risk patients with advanced stage FL. ${ }^{44}$

Because of the long natural history of FL, lack of survival benefit in most studies, and concern regarding the development of secondary malignancies, autologous HSCT for highrisk patients with FL in first CR remains investigational.

\section{Allogeneic transplantation Myeloablative regimens}

Several studies have suggested that allogeneic transplantation for FL may improve the DFS when compared with autologous transplantation because of the presence of the GVL effect. The probability of relapse after allogeneic transplantation has ranged from $10 \%$ to $15 \%{ }^{26}$ In the past, the low relapse rate observed after allogeneic transplantation has not translated into an improvement in OS because of the high TRM associated with the use of HDT (Table 2). ${ }^{26,46}$ For example, in a study of 113 patients published by the International Bone Marrow Transplant Registry in 1998, the probability of DFS 
Table 2 Allogeneic hematopoietic stem cell transplantation for follicular lymphomas: Myeloablative conditioning regimen

\begin{tabular}{|c|c|c|c|c|c|c|c|}
\hline Trial & $\mathbf{N}^{\mathrm{a}}$ & Preparatory regimen & Follow-up (months) & EFS/PFS (\%) & OS (\%) & NRM (\%) & Relapse (\%) \\
\hline \multirow[t]{2}{*}{ van Besien 26} & 113 & TBI $84 \%$ & 25 & 49 & 49 & 40 & 16 \\
\hline & & Non-TBI $16 \%$ & & & & & \\
\hline Kuruvilla ${ }^{47}$ & 37 & $\mathrm{Bu} / \mathrm{Cy}$ & 65 & 79 & 76 & 16 & 3 \\
\hline
\end{tabular}

three years after myeloablative allogeneic transplantation was $49 \%$, but the recurrence rate was only $16 \%$. However, the NRM was $40 \% .{ }^{26}$ Factors associated with improved survival were age $<40$ years, improved performance status at the time of transplantation, chemosensitive disease, and use of TBI-based conditioning regimens. ${ }^{26,47}$

In a report by Kuruvilla et al, 37 patients with indolent FL underwent allogeneic HSCT. ${ }^{47}$ With a median follow-up duration of 63.5 months in survivors, the five-year OS was $79 \%$ and TRM was $15 \%$. The median age of patients in this study was only 45 years (range 24-58). ${ }^{47}$

Although data are limited on the use of unrelated versus related donors, a recent analysis from the National Marrow Donor Program included 52 patients with FL who had received myeloablative allogeneic HSCT between 1991 and 2004. The one-year TRM was $42 \%$ and the two-year PFS was $42 \% .{ }^{48}$

\section{Allogeneic versus autologous transplantation in FL}

Most of the published studies comparing allogeneic and autologous transplantation are retrospective or registrybased (Table 3). A prospective study by the Bone Marrow Transplant-Clinical Trials Network was closed early due to slow accrual, thus highlighting a commonly encountered problem in conducting prospective studies. ${ }^{49}$
Van Besien et al reported on 904 patients who had undergone HSCT for FL. ${ }^{19}$ A total of 176 patients had received allogeneic transplants, 131 patients had received purged autologous transplants, and 597 patients had received unpurged autologous transplants. Five-year TRM rates were $30 \%, 14 \%$, and $8 \%$, and five-year recurrence rates were $21 \%$, $43 \%$, and $58 \%$ after allotransplantation, purged autotransplantation, and unpurged autotransplantation, respectively. In multivariate analyses, allotransplantation had higher TRM and lower disease recurrence. Purged autotransplantation had a 26\% lower recurrence risk than unpurged autotransplantation. However, five-year probabilities of survival were similar in the three groups $(51 \%, 62 \%$, and $55 \%$ after allogeneic, purged autotransplantation, and unpurged autotransplantation, respectively). Advanced age, prolonged interval from diagnosis to transplantation, high lactate dehydrogenase, refractory disease, bone marrow involvement, low performance scores, and transplantation between 1990 and 1993 were associated with adverse outcomes. TBI use was associated with higher TRM but lower recurrence. No association existed between acute or chronic GVHD disease and recurrence after allotransplantation. ${ }^{19}$

In a retrospective study, Verdonck et al compared allogeneic and autologous transplantation in 28 patients with advanced low-grade lymphoma. The probability of disease

Table 3 Myeloablative allogeneic versus autologous hematopoietic stem cell transplantation

\begin{tabular}{|c|c|c|c|c|c|c|c|}
\hline Trial & $\begin{array}{l}\text { Preparatory } \\
\text { regimen }\end{array}$ & $\mathbf{N}$ & $\begin{array}{l}\text { Follow-up } \\
\text { (months) }\end{array}$ & EFS/PFS (\%) & OS (\%) & NRM (\%) & Relapse (\%) \\
\hline \multirow[t]{3}{*}{ Van Besien ${ }^{19}$} & Auto unpurged & 597 & 41 & 31 & 55 & 8 & 58 \\
\hline & Auto purged & $|3|$ & 49 & 39 & 62 & 14 & 43 \\
\hline & Allo & 176 & 36 & 45 & 51 & 30 & 21 \\
\hline \multirow[t]{2}{*}{ Verdonck $^{50}$} & Auto & $18^{a}$ & - & 22 & same & 0 & 83 \\
\hline & Allo & $10^{\mathrm{a}}$ & 41 & 68 & & 3 patients & 0 \\
\hline \multirow[t]{3}{*}{ Hosing ${ }^{20}$} & Auto & 68 & 71 & 17 & 34 & 6 & 74 \\
\hline & Allo & 44 & 53 & 45 & 49 & 34 & 19 \\
\hline & & & & & & & $(P=0.003)$ \\
\hline \multirow[t]{3}{*}{ Stein ${ }^{46}$} & Auto & 36 & - & 71 & 56 & - & - \\
\hline & Allo & 15 & - & 64 & 15 & 46 & - \\
\hline & & & & $(P=0.049)$ & $(P=0.012)$ & & \\
\hline \multirow[t]{3}{*}{ Ingram 24} & Auto & 82 & 88 & 56 & 67 & 2 & 43 \\
\hline & Allo & 44 & 34 & 58 & 69 & 20 & 20 \\
\hline & & & & $(P=N S)$ & $(P=N S)$ & $(P=0.00 I)$ & $(P=0.0 \mathrm{I})$ \\
\hline
\end{tabular}

ancluded other low-grade histologies.

Abbreviations: Auto, autologous; allo, allogeneic; PFS, progression-free survival; EFS, event-free survival; OS, overall survival; NRM, nonrelapse mortality; TBI, total body irradiation; NS, not significant; Bu/Cy, busulfan/cyclophosphamide. 
progression among patients who had undergone allogeneic HSCT was $0 \%$ versus $83 \%$ for autologous recipients at 41 months of follow-up $(P=0.002)$. PFS was $68 \%$ versus $22 \%$ at two years $(P=0.049) .{ }^{50}$

Hosing et al retrospectively compared the outcomes of HDT allogeneic versus autologous HSCT in patients with refractory or recurrent indolent NHL. ${ }^{20}$ Of 112 patients, 68 patients had undergone autologous HSCT and 44 had undergone allogeneic transplantation. In the allogeneic HSCT group, the median follow-up time was 53 months (range 21-113), and the OS and DFS rates were $49 \%$ and $45 \%$, respectively. After a median follow-up time of 71 months (range 22-109) in the autologous HSCT group, the OS and DFS rates were $34 \%$ and $17 \%$, respectively. The probability of disease progression was significantly higher in the autologous HSCT group than it was in the allogeneic HSCT group (74\% versus $19 \% ; P=0.003) .{ }^{20}$

Ingram et al analyzed 126 patients with relapsed advanced-stage FL who received BCNU, cytarabine, etoposide, melphalan (BEAM)-alemtuzumab allogeneic HSCT $(n=44)$ or BEAM-autologous HSCT (BEAM-auto, $n=82) .{ }^{24}$ The allogeneic group had a younger median age but had received a higher median number of therapies pretransplant $(P=0.015)$ than had the autologous group. The allogeneic group had a higher NRM than did the autologous group at one year $(20 \%$ versus $2 \% ; P=0.001)$. Older age and heavy pretreatment were associated with a high NRM and poor survival in the allogeneic group. Noted again was a significantly lower relapse rate $(20 \%$ versus $43 \% ; P=0.01)$ at three years in the allogeneic group, with no relapses after two years compared with a continued pattern of relapse in the autologous group. No difference in OS or DFS was identified at three years, whereas a plateau in OS and DFS with crossing of the survival curves in favor of the allogeneic group was observed. ${ }^{24}$

Using the European Bone Marrow Transplant registry, Peniket et al analyzed 1185 allogeneic transplants for lymphoma reported to the registry between 1982 and 1998 and compared the results with those of 14,687 autologous procedures performed over the same period. ${ }^{51}$ Two hundred and thirty-one patients had low-grade histology, and actuarial OS four years after transplantation was 51\% for this subgroup. These outcomes are relatively poor because of the high procedure-related mortality. Multivariate analysis showed that status at transplantation significantly affected outcome. A matched analysis was performed; for all categories of lymphoma, OS was better for autologous than for allogeneic transplantation, and the relapse rate was lower in the allogeneic group..$^{51}$

\section{Allogeneic nonmyeloablative or reduced- intensity transplantation for FL}

Preliminary results using nonmyeloablative preparative regimens for allogeneic transplantation have been very encouraging, with less than 15\% TRM (Table 4). ${ }^{52-56}$ One of the first reports was published by Khouri et al who treated 20 patients having indolent histology NHL with nonmyeloablative allogeneic HSCT. The day 100 mortality was $10 \%$ and, after a median follow-up duration of 21 months (range 5-46), the actuarial probability of being alive and in remission was $84 \%$ (95\% confidence interval [CI], 53\%-84\%). ${ }^{21}$ In a follow-up report on 47 patients with FL who had received a conditioning regimen of fludarabine, cyclophosphamide, and rituximab followed by allogeneic HSCT, all patients experienced CR posttransplant and only two relapsed..$^{25}$ With a median follow-up time of 60 months (range 19-94), the estimated OS and PFS rates of that study were $85 \%$ and $83 \%$, respectively. The incidence of Grade II-IV acute GVHD was only $11 \% .^{25}$

Rezvani et al studied 62 patients with indolent or transformed NHL who had received allogeneic HSCT from related $(\mathrm{n}=34)$ or unrelated $(\mathrm{n}=28)$ donors after nonmyeloablative conditioning using 2 Gray of TBI \pm fludarabine. ${ }^{52}$ Of the

Table 4 Allogeneic hematopoietic stem cell transplantation for follicular lymphomas: Reduced intensity and/or nonmyeloablative conditioning regimen

\begin{tabular}{|c|c|c|c|c|c|c|c|}
\hline Trial & $\mathbf{N}$ & $\begin{array}{l}\text { Preparatory } \\
\text { regimen }\end{array}$ & $\begin{array}{l}\text { Follow-up } \\
\text { (months) }\end{array}$ & EFS/PFS (\%) & OS (\%) & NRM (\%) & Relapse (\%) \\
\hline Khouri ${ }^{25}$ & $47^{a}$ & $\mathrm{FCR}$ & 36 & 83 & 85 & 15 & 4 \\
\hline Morris $^{57}$ & 41 & FM/Campath & 36 & 65 & 73 & 11 & 44 \\
\hline Kusumi $^{56}$ & $45^{c}$ & Various & 24 & $\begin{array}{l}83 \text { sens } \\
64 \text { res }\end{array}$ & 79 & 18 & 2 \\
\hline Robinson ${ }^{55}$ & $52^{c}$ & Various & 10 & 54 & 65 & 31 & 21 \\
\hline Rezvani ${ }^{52}$ & $62^{\mathrm{b}}$ & $\mathrm{TBI} \pm \mathrm{Flu}$ & 37 & 43 & 52 & 23 & 14 \\
\hline
\end{tabular}

Notes: aPatients with follicular lymphoma; bincluded patients with transformed follicular lymphoma; 'included other low grade histologies.

Abbreviations: FCR, fludarabine, cyclophosphamide, rituximab; FM, fludarabine/melphalan; PFS, progression-free survival; EFS, event-free survival; OS, overall survival; NRM, non-relapse mortality; TBI, total body irradiation; NS, not significant; HR, hazards ratio. 
62 patients, 54 had FL. Sixteen patients had histologic transformation before HSCT, and median follow-up time after transplant was 36.6 months. At three years, the estimated OS and PFS rates were $52 \%$ and $43 \%$, respectively, for patients with indolent disease and $21 \%$ and $18 \%$, respectively, for patients with transformed disease. Their study reported that the incidences of Grade II-IV acute GVHD and extensive chronic GVHD were $63 \%$ and 47\%, respectively. Among survivors, the median Karnofsky performance status at last follow-up was $85 \%$. It is encouraging that long-term survivors reported good overall functional status. ${ }^{52}$

Robinson et al reported the outcome of RIC allogeneic HSCT for 188 patients with lymphoma from the Working Party Lymphoma of the European Group for Blood and Bone Marrow Transplantation. ${ }^{55}$ Fifty-two patients had indolent histology. The two-year TRM, OS, and PFS rates for patients with indolent histology were $31 \%, 65 \%$, and $54 \%$, respectively. Probability of disease recurrence was $21 \%$ at two years. For the entire group (including all histologies), age greater than 50 years was a risk factor for increased TRM. Refractory disease at transplant was associated with an increased risk of progression and poor OS. ${ }^{55}$

Kusumi et al conducted a nationwide survey of 112 adult Japanese patients who had undergone RIC allogeneic HSCT for NHL. ${ }^{56}$ Included in the study were 45 patients with indolent histology. Cumulative incidences of Grade II-IV acute GVHD and chronic GVHD were $49 \%$ and $59 \%$, respectively, for the entire group. With a median follow-up of 23.9 months, three-year OS was $81 \%$ in patients with FL. For patients with indolent histology, three-year PFS was $83 \%$ for chemosensitive patients and $64 \%$ for chemorefractory patients. ${ }^{56}$
Morris et al reported the outcomes of RIC allogeneic HSCT for NHL in 88 patients, 41 of whom had low-grade histology. ${ }^{57,58}$ With a median follow-up of 36 months (range 18-60 months), the actuarial OS rates at three years were $73 \%$. The 100-day and three-year TRM rates were 2\% and $11 \%$, respectively. The actuarial current PFS rate at three years, including the rate for patients who achieved remission after donor lymphocyte infusion was $65 \%$. Patients with low-grade NHL and chronic lymphocytic leukemia achieved excellent PFS with extremely low TRM and GVHD, even when matched related donors were unavailable. ${ }^{57,58}$

\section{Myeloablative versus reduced-intensity conditioning regimens}

The studies discussed in this section are also retrospective studies and differ in their patient characteristics. In general, patients who received myeloablative regimens were younger, had good performance status, had fewer comorbidities, and were more likely to have refractory disease (Table 5).

Khouri et al compared allogeneic transplantation for indolent NHL using HDT versus nonmyeloablative regimens in patients who had chemosensitive disease at the time of transplantation. ${ }^{59} \mathrm{OS}$ and DFS were $100 \%$ for the nonmyeloablative arm versus $36 \%$ for the HDT arm $(P=0.004)$. The median follow-up time in that study was 30 months (range 2-79 months). ${ }^{59}$

In a retrospective analysis of 208 transplants reported to the Center for International Blood and Marrow Transplant Research between 1997 and 2002, Hari et al compared traditional myeloablative conditioning regimens with RIC for FL. ${ }^{54}$ Conditioning regimens were categorized as

Table 5 Allogeneic hematopoietic stem cell transplantation for follicular lymphoma: Reduced intensity and/or nonmyeloablative versus myeloablative conditioning regimen

\begin{tabular}{|c|c|c|c|c|c|c|c|}
\hline Trial & $\mathbf{N}^{\mathrm{a}}$ & $\begin{array}{l}\text { Preparatory } \\
\text { regimen }\end{array}$ & $\begin{array}{l}\text { Follow-up } \\
\text { (months) }\end{array}$ & EFS/PFS (\%) & OS (\%) & NRM (\%) & Relapse (\%) \\
\hline \multirow[t]{3}{*}{$\operatorname{Hari}^{54}$} & MA 120 & Various & 50 & 67 & 71 & 23 & 17 \\
\hline & RIC 88 & & 35 & 55 & 62 & 23 & \\
\hline & & & & $(P=0.07)$ & $(P=0.15)$ & $(P=N S)$ & \\
\hline \multirow[t]{3}{*}{ Rodriguez $^{53}$} & MA 18 & $\mathrm{Cy} / \mathrm{TBI}$ or $\mathrm{Bu} / \mathrm{cy}$ & 69 & 50 & 56 & 38 & 19 \\
\hline & RIC 16 & $\mathrm{FM}$ & 20 & 49 & 68 & 28 & \\
\hline & & & & $(P=0.56)$ & $(P=0.83)$ & $(P=0.4)$ & \\
\hline \multirow[t]{3}{*}{ Sorror ${ }^{60}$} & MA 27 & $\mathrm{Cy} / \mathrm{TBI}$ or $\mathrm{Bu} / \mathrm{cy}$ & 61 & HR MA versus & HR MA versus & - & - \\
\hline & RIC I4 & $\mathrm{TBI} \pm \mathrm{Flu}$ & 44 & RIC 3.16 & RIC 2.02 & & \\
\hline & & & & $(P=0.02)$ & $(P=0.07)$ & & \\
\hline \multirow[t]{3}{*}{ Avivi $^{61}$} & MA 44 & various & 38 & 43 & 47 & 37 & same \\
\hline & RIC 87 & & 34 & 49 & 53 & 33 & \\
\hline & & & & $(P=N S)$ & $(P=N S)$ & $(P=N S)$ & \\
\hline
\end{tabular}

Notes: ${ }^{a}$ Patients with follicular lymphoma; bincluded patients with transformed follicular lymphoma; included other low grade histologies.

Abbreviations: MA, myeloablative; RIC, reduced intensity conditioning; PFS, progression-free survival; EFS, event-free survival; OS, overall survival; NRM, nonrelapse mortality; NS, not significant; HR, hazards ratio; Cy/TBI, cyclophosphamide/total body irradiation; Bu/Cy, busulfan/cyclophosphamide; FM, fludarabine/melphalan; Flu, fludarabine. 
myeloablative $(n=120)$ or RIC $(n=88)$. Patients who had received RIC were older and had had a longer time interval from diagnosis to transplant. Median follow-up of survivors was 50 months (range 4-96) after myeloablative conditioning versus 35 months (range 4-82) after RIC $(P<0.001)$. Surprisingly, the OS, PFS, and TRM did not differ between the two groups. At three years, OS rates for the myeloablative and RIC cohorts were $71 \%$ and $62 \%(P=0.15)$ and PFS rates were $67 \%$ and $55 \%(P=0.07)$, respectively. Lower performance score and resistance to chemotherapy were associated with higher TRM and lower OS and PFS rates. On multivariate analysis, an increased risk of lymphoma progression after RIC was observed (relative risk $[R R]=2.97$; $P=0.04){ }^{54}$

Rodriguez et al performed a retrospective analysis of 88 patients who had been conditioned with conventional myeloablative regimens $(n=48)$ or RIC $(n=40) .{ }^{53}$ Compared with patients who had received myeloablative regimens, those who had received RIC were older, had more often failed autologous transplantation, and had more frequently received peripheral blood and unrelated donor transplants. The relapse rate was significantly lower in the patients receiving myeloablative regimens than it was in those receiving RIC regimens (13\% versus $28 \% ; P=0.05$ ). The one-year transplantationrelated mortality rate was $33 \%$ for myeloablative and $28 \%$ for RIC regimens $(P=0.40)$. Kaplan-Meier two-year OS and PFS were $52 \%$ and $46 \%$ for myeloablative versus $53 \%$ and $40 \%$ for RIC regimens ( $P=$ not significant). Factors associated with improved survival were chemosensitive disease at transplantation $(\mathrm{RR}=57 \% ; P=0.05)$ and absence of prior autologous HSCT $(\mathrm{RR}=37 \% ; P=0.002) .{ }^{53}$

Sorror et al stratified outcomes using the hematopoietic cell transplantation-specific comorbidity index. ${ }^{60}$ Patients in the nonmyeloablative group were older, had more previous treatment and more comorbidities, more frequently had unrelated donors, and more often had malignancy in remission than did patients in the myeloablative group. After HSCT, patients without comorbidities both in the nonmyeloablative and myeloablative cohorts had comparable NRM, OS, and PFS. Patients with comorbidities experienced lower NRM and better OS after nonmyeloablative conditioning. Nonmyeloablative transplant recipients with comorbidities had favorable adjusted PFS compared with patients in the myeloablative group. ${ }^{60}$

Avivi et al analyzed the outcomes of patients with FL treated with matched unrelated allogeneic HSCT.${ }^{61} \mathrm{~A}$ total of 131 patients had been treated with RIC $(n=87)$ or conventional myeloablative $(n=44)$ regimens between 2000 and 2005. RIC recipients were significantly older, with a longer interval from diagnosis to transplant, and had failed a previous autograft more often than patients treated with a conventional myeloablative regimen. NRM was $30 \%$ at one year. After a median follow-up of 36 months, the three-year PFS was $47 \%$. The three-year OS rate for the whole series was $51 \%$. On multivariate analysis, RIC regimens were associated with a lower NRM and a significantly longer PFS and OS. ${ }^{61}$

In recent years, RIC has become the standard for allogeneic HSCT in FL and appears to result in similar longterm outcomes. Although DFS is similar to myeloablative conditioning, some studies have shown an increased risk of late disease progression after RIC, which is concerning. Although some of these patients may be induced back into remission with the judicious use of donor lymphocyte infusion, there is a risk of inducing severe GVHD with repeated donor lymphocyte infusions. Therefore, the impact of dose reduction on relapse and survival needs additional study. Use of monoclonal antibodies and radioimmunoconjugates in the allogeneic transplant regimens may also affect outcomes, and studies are ongoing. ${ }^{25,62}$

\section{Discussion}

Selected patients with FL may benefit from HSCT, but it is still uncertain which subset of patients may receive the most benefit. Patients with relapsed and chemosensitive FL should be considered for autologous HSCT because several Phase II studies have shown survival advantage and prolonged remissions. ${ }^{28,33}$ Although data are limited, it is reasonable to expect that the addition of monoclonal antibodies like rituximab during stem cell collection for in vivo purging will reduce tumor contamination of the graft and therefore reduce relapse rates. The role of autologous HSCT for FL in the rituximab era has been questioned by some, but preliminary data suggest that HDT-autologous HSCT is still beneficial in patients with FL who relapse following rituximab-containing regimens. ${ }^{45,63}$ Similarly, incorporation of monoclonal antibodies or radioimmunoconjugates during HDT is feasible and may improve the long-term outcomes, and studies are ongoing. Concern remains, however, regarding the increased risk of s-MDS/s-AML observed by most investigators. The patients who appear to benefit most from this strategy are those with chemosensitive disease, who have received less than three chemotherapy regimens, and do not have high-risk FL international prognostic index scores. ${ }^{64}$ Addition of TBI has been shown to increase the risk of developing s-MDS/s-AML in some studies and 
therefore non-TBI-based regimens should be used when feasible. ${ }^{33}$ Maintenance therapy after transplantation may also be considered in a subset of patients, although data is lacking in those with FL.

Despite four large randomized studies comparing upfront autologous HSCT with standard chemotherapy in FL, no survival advantage was noted for the HSCT arms. Two of these studies, GOELAMS ${ }^{9}$ and GLSG, ${ }^{32}$ reported a longer PFS in the transplant arms without survival advantage in GOELAMS, and the OS is not yet reported for GLSG. Only one upfront randomized study included rituximab in the treatment regimen, and that study also failed to show a survival advantage for HSCT despite longer EFS. ${ }^{43}$ Thus, upfront autologous HSCT for FL should only be done in clinical trials.

Allogeneic HSCT has been shown to cure patients with FL. Allogeneic transplants should be offered to patients who are young, have a matched donor, and are beyond first CR. Myeloablative regimens are associated with significant TRM. Use of nonmyeloablative or RIC regimens appears very promising, but more studies and longer follow-up is needed before definite treatment recommendations can be made. There is concern regarding higher relapse rates after nonmyeloablative or RIC regimens. At our institution we offer allogeneic HSCT to younger FL patients who have good performance status, are beyond first remission, and who have a matched human leukocyte antigen donor available. These patients should be enrolled in clinical trials whenever possible.

\section{Disclosures}

The author reports no conflict of interest in this work.

\section{References}

1. Armitage JO, Weisenburger DD. New approach to classifying nonHodgkin's lymphomas: Clinical features of the major histologic subtypes. Non-Hodgkin's Lymphoma Classification Project. J Clin Oncol. 1998;16(8):2780-2795.

2. Johnson PW, Rohatiner AZ, Whelan JS, et al. Patterns of survival in patients with recurrent follicular lymphoma: A 20-year study from a single center. J Clin Oncol. 1995;13(1):140-147.

3. Horning SJ. Follicular lymphoma: Have we made any progress? Ann Oncol. 2000;11 Suppl 1:23-27.

4. Fisher RI, LeBlanc M, Press OW, Maloney DG, Unger JM, Miller TP. New treatment options have changed the survival of patients with follicular lymphoma. J Clin Oncol. 2005;23(33):8447-8452.

5. Swenson WT, Wooldridge JE, Lynch CF, Forman-Hoffman VL, Chrischilles E, Link BK. Improved survival of follicular lymphoma patients in the United States. J Clin Oncol. 2005;23(22):5019-5026.

6. Czuczman MS, Weaver R, Alkuzweny B, Berlfein J, Grillo-Lopez AJ. Prolonged clinical and molecular remission in patients with low-grade or follicular non-Hodgkin's lymphoma treated with rituximab plus CHOP chemotherapy: 9-year follow-up. J Clin Oncol. 2004;22(23): 4711-4716.

7. Vidal L, Gafter-Gvili A, Leibovici L, Shpilberg O. Rituximab as maintenance therapy for patients with follicular lymphoma. Cochrane Database Syst Rev. 2009(2):CD006552.
8. Morschhauser F, Radford J, Van Hoof A, et al. Phase III trial of consolidation therapy with yttrium-90-ibritumomab tiuxetan compared with no additional therapy after first remission in advanced follicular lymphoma. J Clin Oncol. 2008;26(32):5156-5164.

9. Gyan E, Foussard C, Bertrand P, et al. High-dose therapy followed by autologous purged stem cell transplantation and doxorubicin-based chemotherapy in patients with advanced follicular lymphoma: A randomized multicenter study by the GOELAMS with final results after a median follow-up of 9 years. Blood. 2009;113(5):995-1001.

10. Gopal AK, Gooley TA, Maloney DG, et al. High-dose radioimmunotherapy versus conventional high-dose therapy and autologous hematopoietic stem cell transplantation for relapsed follicular non-Hodgkin lymphoma: A multivariable cohort analysis. Blood. 2003;102(7):2351-2357.

11. Tarella C, Zanni M, Magni M, et al. Rituximab improves the efficacy of high-dose chemotherapy with autograft for high-risk follicular and diffuse large B-cell lymphoma: A multicenter Gruppo Italiano Terapie Innnovative nei linfomi survey. J Clin Oncol. 2008; 26(19):3166-3175.

12. Voso MT, Pantel G, Weis M, et al. In vivo depletion of B cells using a combination of high-dose cytosine arabinoside/mitoxantrone and rituximab for autografting in patients with non-Hodgkin's lymphoma. Br J Haematol. 2000;109(4):729-735.

13. Horowitz MM, Gale RP, Sondel PM, et al. Graft-versus-leukemia reactions after bone marrow transplantation. Blood. 1990;75(3): $555-562$.

14. Gale RP, Champlin RE. How does bone-marrow transplantation cure leukaemia? Lancet. 1984;2(8393):28-30.

15. Weiden PL, Flournoy N, Sanders JE, Sullivan KM, Thomas ED. Antileukemic effect of graft-versus-host disease contributes to improved survival after allogeneic marrow transplantation. Transplant Proc. 1981;13(1 Pt 1):248-251.

16. van Besien KW, de Lima M, Giralt SA, et al. Management of lymphoma recurrence after allogeneic transplantation: The relevance of graft-versus-lymphoma effect. Bone Marrow Transplant. 1997;19(10):977-982.

17. Rondon G, Giralt S, Huh Y, et al. Graft-versus-leukemia effect after allogeneic bone marrow transplantation for chronic lymphocytic leukemia. Bone Marrow Transplant. 1996;18(3):669-672.

18. Kolb HJ, Schattenberg A, Goldman JM, et al. Graft-versus-leukemia effect of donor lymphocyte transfusions in marrow grafted patients. Blood. 1995;86(5):2041-2050.

19. van Besien K, Loberiza FR Jr, Bajorunaite R, et al. Comparison of autologous and allogeneic hematopoietic stem cell transplantation for follicular lymphoma. Blood. 2003;102(10):3521-3529.

20. Hosing C, Saliba RM, McLaughlin P, et al. Long-term results favor allogeneic over autologous hematopoietic stem cell transplantation in patients with refractory or recurrent indolent non-Hodgkin's lymphoma. Ann Oncol. 2003;14(5):737-744.

21. Khouri IF, Saliba RM, Giralt SA, et al. Nonablative allogeneic hematopoietic transplantation as adoptive immunotherapy for indolent lymphoma: Low incidence of toxicity, acute graft-versus-host disease, and treatment-related mortality. Blood. 2001;98(13):3595-3599.

22. Antin JH, Ferrara JL. Cytokine dysregulation and acute graft-versushost disease. Blood. 1992;80(12):2964-2968.

23. Hill GR, Crawford JM, Cooke KR, Brinson YS, Pan L, Ferrara JL. Total body irradiation and acute graft-versus-host disease: The role of gastrointestinal damage and inflammatory cytokines. Blood. 1997; 90(8):3204-3213.

24. Ingram W, Devereux S, Das-Gupta EP, et al. Outcome of BEAMautologous and BEAM-alemtuzumab allogeneic transplantation in relapsed advanced stage follicular lymphoma. Br J Haematol. 2008;141(2):235-243.

25. Khouri IF, McLaughlin P, Saliba RM, et al. Eight-year experience with allogeneic stem cell transplantation for relapsed follicular lymphoma after nonmyeloablative conditioning with fludarabine, cyclophosphamide, and rituximab. Blood. 2008;111(12):5530-5536. 
26. van Besien K, Sobocinski KA, Rowlings PA, et al. Allogeneic bone marrow transplantation for low-grade lymphoma. Blood. 1998;92(5):1832-1836.

27. Surbone A, Armitage JO, Gale RP. Autotransplantation in lymphoma: Better therapy or healthier patients? Ann Intern Med. 1991;114(12):1059-1060.

28. Rohatiner AZ, Nadler L, Davies AJ, et al. Myeloablative therapy with autologous bone marrow transplantation for follicular lymphoma at the time of second or subsequent remission: Long-term follow-up. J Clin Oncol. 2007;25(18):2554-2559.

29. Freedman AS, Neuberg D, Mauch P, et al. Long-term follow-up of autologous bone marrow transplantation in patients with relapsed follicular lymphoma. Blood. 1999;94(10):3325-3333.

30. Bierman PJ, Vose JM, Anderson JR, Bishop MR, Kessinger A, Armitage JO. High-dose therapy with autologous hematopoietic rescue for follicular low-grade non-Hodgkin's lymphoma. J Clin Oncol. 1997; 15(2):445-450.

31. Freedman AS, Gribben JG, Neuberg D, et al. High-dose therapy and autologous bone marrow transplantation in patients with follicular lymphoma during first remission. Blood. 1996;88(7): 2780-2786.

32. Lenz G, Dreyling M, Schiegnitz E, et al. Myeloablative radiochemotherapy followed by autologous stem cell transplantation in first remission prolongs progression-free survival in follicular lymphoma: Results of a prospective, randomized trial of the German Low-Grade Lymphoma Study Group. Blood. 2004;104(9):2667-2674.

33. Montoto S, Canals C, Rohatiner AZ, et al. Long-term follow-up of high-dose treatment with autologous haematopoietic progenitor cell support in 693 patients with follicular lymphoma: An EBMT registry study. Leukemia. 2007;21(11):2324-2331.

34. Schouten HC, Kvaloy S, Sydes M, Qian W, Fayers PM. The CUP trial: A randomized study analyzing the efficacy of high dose therapy and purging in low-grade non-Hodgkin's lymphoma (NHL). Ann Oncol. 2000;11 Suppl 1:91-94.

35. Schouten HC, Qian W, Kvaloy S, et al. High-dose therapy improves progression-free survival and survival in relapsed follicular nonHodgkin's lymphoma: Results from the randomized European CUP trial. J Clin Oncol. 2003;21(21):3918-3927.

36. Apostolidis J, Gupta RK, Grenzelias D, et al. High-dose therapy with autologous bone marrow support as consolidation of remission in follicular lymphoma: Long-term clinical and molecular follow-up. J Clin Oncol. 2000;18(3):527-536.

37. Cao TM, Horning S, Negrin RS, et al. High-dose therapy and autologous hematopoietic-cell transplantation for follicular lymphoma beyond first remission: The Stanford University experience. Biol Blood Marrow Transplant. 2001;7(5):294-301.

38. Arcaini L, Montanari F, Alessandrino EP, et al. Immunochemotherapy with in vivo purging and autotransplant induces long clinical and molecular remission in advanced relapsed and refractory follicular lymphoma. Ann Oncol. 2008;19(7):1331-1335.

39. Arcaini L, Orlandi E, Alessandrino EP, et al. A model of in vivo purging with Rituximab and high-dose AraC in follicular and mantle cell lymphoma. Bone Marrow Transplant. 2004;34(2):175-179.

40. Sebban C, Mounier N, Brousse N, et al. Standard chemotherapy with interferon compared with CHOP followed by high-dose therapy with autologous stem cell transplantation in untreated patients with advanced follicular lymphoma: The GELF-94 randomized study from the Groupe d'Etude des Lymphomes de 1'Adulte (GELA). Blood. 2006;108(8):2540-2544.

41. Sebban C, Brice P, Delarue R, et al. Impact of rituximab and/ or high-dose therapy with autotransplant at time of relapse in patients with follicular lymphoma: A GELA study. J Clin Oncol. 2008;26(21):3614-3620.

42. Rohatiner AZ, Freedman A, Nadler L, Lim J, Lister TA. Myeloablative therapy with autologous bone marrow transplantation as consolidation therapy for follicular lymphoma. Ann Oncol. 1994;5 Suppl 2: 143-146.
43. Ladetto M, De Marco F, Benedetti F, et al. Prospective, multicenter randomized GITMO/IIL trial comparing intensive (R-HDS) versus conventional (CHOP-R) chemoimmunotherapy in high-risk follicular lymphoma at diagnosis: The superior disease control of R-HDS does not translate into an overall survival advantage. Blood. 2008;111(8):4004-4013.

44. Buske C, Hoster E, Dreyling M, et al. Rituximab in combination with CHOP in patients with follicular lymphoma: Analysis of treatment outcome of 552 patients treated in a randomized trial of the German Low Grade Lymphoma Study Group (GLSG) after a follow up of 58 months. Blood. 2008;112:901.

45. Hiddemann W, Buske, C, Kneba, M, et al. Autologous stem cell transplantation after myeloablative therapy in first remission may be beneficial in patients with advanced stage follicular lymphoma after front-line therapy with R-CHOP. An analysis of two consecutive studies of the German Low Grade Lymphoma Study Group (GLSG). Blood. 2008;112:286-287.

46. Stein RS, Greer JP, Goodman S, Kallianpur A, Ahmed MS, Wolff SN. High-dose therapy with autologous or allogeneic transplantation as salvage therapy for small cleaved cell lymphoma of follicular center cell origin. Bone Marrow Transplant. 1999;23(3):227-233.

47. Kuruvilla J, Pond G, Tsang R, Gupta V, Lipton JH, Messner HA. Favorable overall survival with fully myeloablative allogeneic stem cell transplantation for follicular lymphoma. Biol Blood Marrow Transplant. 2008;14(7):775-782.

48. van Besien K, Carreras J, Bierman PJ, et al. Unrelated donor hematopoietic cell transplantation for non-Hodgkin lymphoma: Longterm outcomes. Biol Blood Marrow Transplant. 2009;15(5):554-563.

49. Laport GCB, Tomblyn MR, Kahl BS, et al: on behalf of the Blood and Marrow Transplant Clinical Trials Network (BMT CTN). Autologous versus reduced-intensity allogeneic hematopoietic cell transplantation for patients with follicular non-Hodgkins lymphoma (FL) beyond first complete response or first partial response. J Clin Oncol. 2008;26:382a.

50. Verdonck LF, Dekker AW, Lokhorst HM, Petersen EJ, Nieuwenhuis HK. Allogeneic versus autologous bone marrow transplantation for refractory and recurrent low-grade non-Hodgkin's lymphoma. Blood. 1997;90(10):4201-4205.

51. Peniket AJ, Ruiz de Elvira MC, Taghipour G, et al. An EBMT registry matched study of allogeneic stem cell transplants for lymphoma: Allogeneic transplantation is associated with a lower relapse rate but a higher procedure-related mortality rate than autologous transplantation. Bone Marrow Transplant. 2003;31(8):667-668.

52. Rezvani AR, Storer B, Maris M, et al. Nonmyeloablative allogeneic hematopoietic cell transplantation in relapsed, refractory, and transformed indolent non-Hodgkin's lymphoma. J Clin Oncol. 2008;26(2):211-217.

53. Rodriguez R, Nademanee A, Ruel N, et al. Comparison of reducedintensity and conventional myeloablative regimens for allogeneic transplantation in non-Hodgkin's lymphoma. Biol Blood Marrow Transplant. 2006;12(12):1326-1334.

54. Hari P, Carreras J, Zhang MJ, et al. Allogeneic transplants in follicular lymphoma: Higher risk of disease progression after reduced-intensity compared to myeloablative conditioning. Biol Blood Marrow Transplant. 2008;14(2):236-245.

55. Robinson SP, Goldstone AH, Mackinnon S, et al. Chemoresistant or aggressive lymphoma predicts for a poor outcome following reducedintensity allogeneic progenitor cell transplantation: An analysis from the Lymphoma Working Party of the European Group for Blood and Bone Marrow Transplantation. Blood. 2002;100(13):4310-4316.

56. Kusumi E, Kami M, Kanda Y, et al. Reduced-intensity hematopoietic stemcell transplantation for malignant lymphoma: A retrospective survey of 112 adult patients in Japan. Bone Marrow Transplant. 2005;36(3):205-213.

57. Morris E, Thomson K, Craddock C, et al. Outcomes after alemtuzumabcontaining reduced-intensity allogeneic transplantation regimen for relapsed and refractory non-Hodgkin's lymphoma. Blood. 2004;104(13):3865-3871.

58. Morris E, Mackinnon S. Outcome following alemtuzumab (CAMPATH-1H)-containing reduced intensity allogeneic transplant regimen for relapsed and refractory non-Hodgkin's lymphoma (NHL). Transfus Apher Sci. 2005;32(1):73-83. 
59. Khouri I, Saliba R, Giralt S. Allogeneic hematopoietic transplantation for indolent lymphomas: Improved outcome with non-myeloablative versus high dose chemotherapy (HDCT) regimens. Blood. 2000; 96:853a.

60. Sorror ML, Storer BE, Maloney DG, Sandmaier BM, Martin PJ, Storb R. Outcomes after allogeneic hematopoietic cell transplantation with nonmyeloablative or myeloablative conditioning regimens for treatment of lymphoma and chronic lymphocytic leukemia. Blood. 2008;111(1):446-452.

61. Avivi I, Montoto S, Canals C, et al. Matched unrelated donor stem cell transplant in 131 patients with follicular lymphoma: An analysis from the Lymphoma Working Party of the European Group for Blood and Marrow Transplantation. Br J Haematol. 2009;147(5):719-728.
62. Bethge WA, Wilbur DS, Sandmaier BM. Radioimmunotherapy as non-myeloablative conditioning for allogeneic marrow transplantation. Leuk Lymphoma. 2006;47(7):1205-1214.

63. Kang TY, Rybicki LA, Bolwell BJ, et al. Effect of prior rituximab on high-dose therapy and autologous stem cell transplantation in follicular lymphoma. Bone Marrow Transplant. 2007;40(10): 973-978.

64. Vose JM, Bierman PJ, Loberiza FR, et al. Long-term outcomes of autologous stem cell transplantation for follicular non-Hodgkin lymphoma: Effect of histological grade and Follicular International Prognostic Index. Biol Blood Marrow Transplant. 2008;14(1): 36-42.

\section{Publish your work in this journal}

Stem Cells and Cloning: Advances and Applications is an international, peer-reviewed, open access journal. Areas of interest in stem cell research include: Embryonic cell stems; Adult stem cells; Blastocysts; Cordblood stem cells; Stem cell transformation and culture; Therapeutic cloning; Umbilical cord blood and bone marrow cells; Laboratory, animal and human therapeutic studies; Philosophical and ethical issues related to stem cell research. This journal is indexed on CAS. The manuscript management system is completely online and includes a quick and fair peer-review system. Visit http://www.dovepress.com/ testimonials.php to read real quotes from published authors.

Submit your manuscript here: http://www.dovepress.com/stem-cells-and-cloning-advances-and-applications-journal 